\title{
Europe develops action plan to address health workforce shortfall
}

Published at www.cmaj.ca on Nov. 12

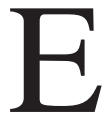
uropean officials have developed an action plan to resolve an anticipated shortfall of one million health workers among the 27 member states of the European Union (EU) by 2020.

The shortfall will include a need for 230000 physicians and as a consequence, about $13.5 \%$ of necessary health care will not be provided, the European Observatory on Health Systems and Policies, states in a report, Investing in Europe's health workforce of tomorrow: Scope for innovation and collaboration, (www.healthworkforce4europe.eu/down loads/Report_PD_Leuven_FINAL.pdf).

The projected workforce shortfall inspired roughly 250 well-heeled health ministers and health administrators to gather in Belgium in September to address four so-called "key issues: the assessment of future health workforce needs; finding the right "mix" of skills in groups of health care workers; creating a supportive working environment; and promoting a "learning culture" to improve patient care and safety.

What emerged is an action plan that organizers hope lays the foundation for better long-term health workforce planning (www.health.belgium.be/eportal /Aboutus/eutrio/health/europehealth workforce/10september/summary_parallel _sessions/index.htm?fodnlang=en).

The plan commits member-states to a series of specific action measures, including:

- "Support for a platform to develop countries' capacity for data collection and analysis, and for policy development and implementation in relation to the health workforce"

- "Structured support for early career workers"

- "Use of structural funds for bridging and retraining professionals" and the possible creation of a health sector council on jobs and skills

- Equivalency in specialist training

- Establishing EU-wide continuining professional development accreditation equivalency using a "competency-based learning framework."

- Use of European "core-curricula for patient safety" in medical education and training programs

Such measures will help address the challenges but they aren't the sole solution, several experts say.

For example, resolving the shortage of health care workers involves more

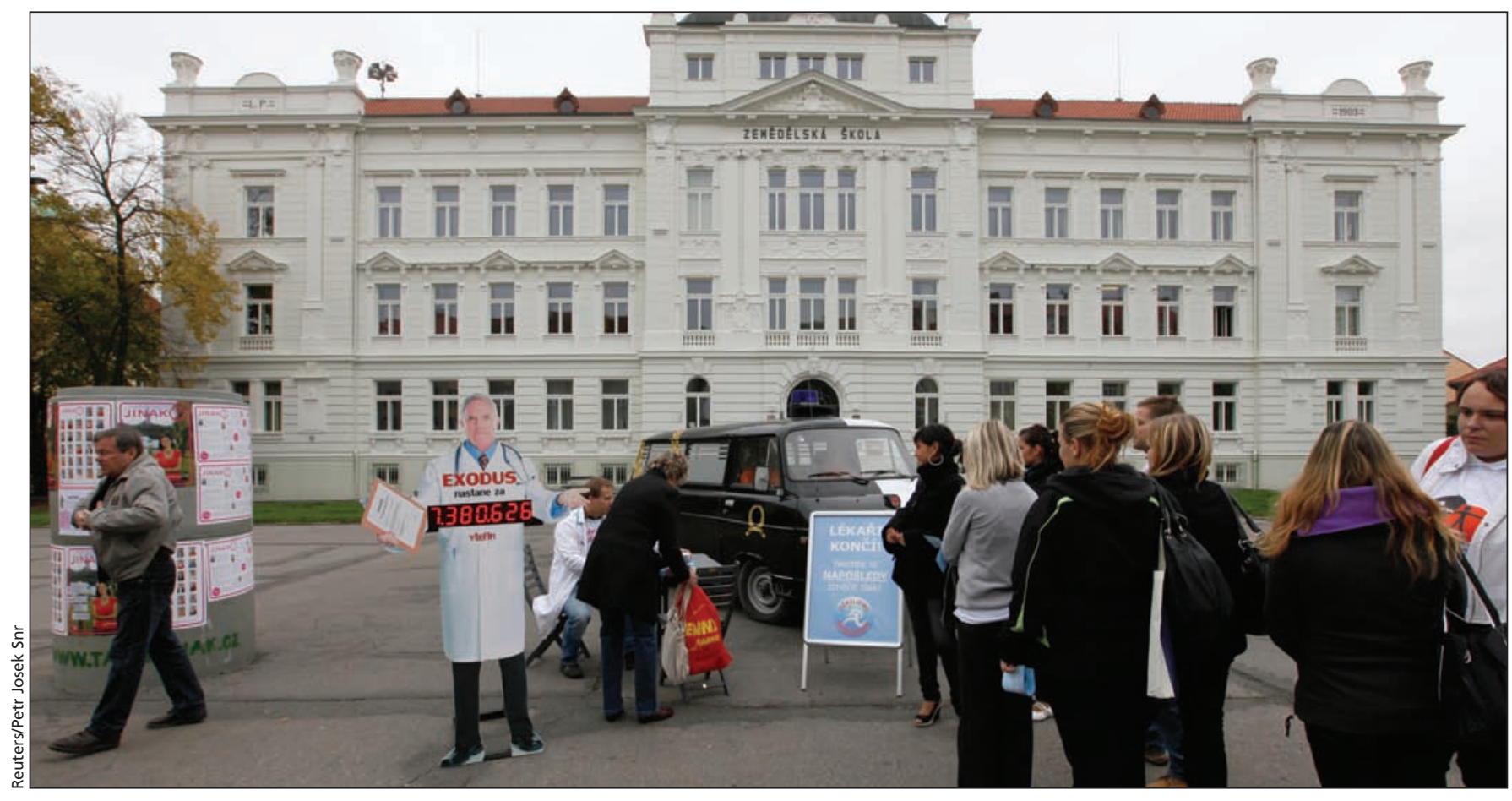

Residents of the town of Tabor in the Czech Republic line up to have their blood pressure measured last month as Czech medical staff protest government austerity measures. Over 3000 hospital doctors have signed a petition threatening to quit by the end of the year and seek work abroad. A figure of "Doctor Exodus" counts down the time until the end of the year. Meanwhile, a new report says Europe will need 230000 more physicians by the year 2020. 
than just a "simplistic" increase in their numbers, says Gilles Dussault, director of the health systems unit at Lisbon's Institute of Hygiene and Tropical Medicine in Lisbon, Portugal.

"It does not take into account numerous factors such as productivity and quality of services," Dussault says. "In Europe, there are great variations in productivity and quality due to differences in the definition of the scope of practice of the various categories of health workers, to work organization, to availability of tools and equipment which augment efficiency, and to competency levels."

"For example, in some countries Sweden, Norway, the UK, France nurses can work autonomously in some clinical environments, and in some cases have more or less extended prescription rights," he adds. "In other countries - Romania, Bulgaria, Hungary - they still perform menial work with little clinical contents. The organization of work varies from strong emphasis on teamwork, on ambulatory care, on coordination between levels of care, to rigid and authoritarian hierarchies, prevalence of hospital care and segmentation of services."

Many jurisdictions are exploring an expansion of responsibilities for nurses and other health care workers as a response to the health human resource shortfall.
But European physicians, like those in Canada and the United States, resist such role revisions, write Marie-Laure Delamaire, a consultant in health and human resources management, and Gaetan Lafortune, a health economist at the Organisation for Economic Cooperation and Development, in a collaborative email. Concerns include "a potential overlap in the scope of practice and a potential loss of their activities, particularly as it relates to the development of nurse practitioners in primary care. They are also concerned about the degree of autonomy and independence of advanced practice nurses, about legal liability in cases of malpractice in the context of teamwork, and also possibly about whether advanced practice nurses have the required skills and expertise to perform some of the new tasks."

Similarly, Europe is a long way from achieving something like a "homogeneous Continuous Professional Development System across member states," writes Dr. Tanya Horsley and a team at Centre for Learning in Practice of the Royal College of Physicians and Surgeons of Canada in an email. But "there are several important initiatives in the EU that are addressing current differences. For example, the European Accreditation Council for Continuing Medical
Education has developed a process by which credit systems in one country can be translated into an EU credit system."

Others say that the patient safety and quality measures urged under the action plan are inadequate.

"The use of validated instruments is a good start to raise awareness, improve culture and promote patient empowerment in the medical field. Of course a lot needs to be done to actually improve patient safety outcomes on the long term in the EU," state Dr. Jean Bacou, project coordinator of the European Network for Patient Safety in an email. "The main priorities are in terms of the organisation of the health care system (including primary care: promoting teamwork and life-long learning for health care professionals, integrated care and patient centeredness. Member States need to invest in this field to really address patient safety issues. One priority would be for each Member State to establish a national quality improvement system (for example, certification system for health care organizations and continuous professional development for health care professionals)." - Tiago Villanueva MD, Lisbon, Portugal

DOI:10.1503/cmaj.109-3708 